\title{
REDUCING CALL BLOCKING PROBABILITY IN HYBRID MULTICAST WAVELENGTH DIVISION MULTIPLEXING NETWORKS USING WEIGHTED ROUTING AND WAVELENGTH ASSIGNMENT ALGORITHM
}

\author{
${ }^{1}$ Pradeep Sankar, D.V. and ${ }^{2}$ K. Muneeswaran \\ ${ }^{1}$ Department of Information Technology, P.S.R., Engineering College, Sivakasi, India \\ ${ }^{2}$ Department of Computer Science and Engineering, Mepco Schlenk Engineering College, Sivakasi, India
}

Received 2013-09-24, Revised 2013-10-10; Accepted 2013-11-13

\begin{abstract}
In the proposed work, the focus is on increasing the available wavelength in the all-optical networks, for which the Routing and Wavelength Assignment plays a vital role. In the earlier reported work, the wavelengths were assigned to the links which covers a set of destinations but not all the intended destinations. However this proposed work, attempts were made to create multiple spanning tree with minimum cost for reaching all the nodes in the network. Also, the delay (associated with transmission and propagation delay) was considered in the proposed work for demonstrating the Quality of Service. Experimentally the proposed algorithm works by means of constructing Multiple Minimum Spanning Trees (MMST) using Mayeda and Seshu algorithm considering the QoS parameter delay and avoids the call blocking probabilities. For the performance measurement, the parameters such as the cost and delay are considered and depending on the application they can be traded-off. The significance of the work lies in the optimization of the wavelength used in the fibre network, which mainly contributes to the backbone of the Internet. The experiments were conducted for the different scenarios of simulated network configuration and found to produce promising results compared to the existing work.
\end{abstract}

Keywords: RWA Algorithms, QOS, WDM Networks, Multicast, MRWA, Greedy Techniques

\section{INTRODUCTION}

Internet leads to be a part of our daily life where people digging for their desired data that require high bandwidth. The promise of convention of the high bandwidth is done by the way of splitting massive transmission bandwidth of optical fiber into multiple channels. A technology like optical network requires to use Wavelength Division Multiplexing (WDM) technique to exploit the optical fibre bandwidth capacity. Here, the bandwidth is distributed in various channels with dissimilar wavelength, whereas each channel works at its peak rate. Across the past technological era, the entry of factors like the Quality of Service (QoS) (Cao and Yu, 2005; Chen et al., 2004) and the differentiated service
(Zhang and Knightly, 1999. Golmie et al., 2000) enhance through variable stages of service performance in the very same network (Fumagalli and Tacca, 2001a; 2001b). The particular issue of Power Aware-Routing and Wavelength Assignment (PA-RWA) is taken care in accommodating a transparent multi-fibre optical network which is scrutinized in the work put forward in (Coiro et al., 2011). Using the concept of reserving one wavelength on each hop which constructs a light path on optical fibre routes in the urge of satisfying the traffic demand based on the granular nature of wavelength is a topic which is under thorough research in the WDM optical networks field. The data channel (optical path) is constructed from one node to another node in the network which is assigned with the very same wavelength all along its path and can Corresponding Author: Pradeep Sankar, D.V., Department of Information Technology, P.S.R., Engineering College, Sivakasi, India 
come across multiple links. The occupation of the same wavelength by the light path might take place due to the absence of wavelength transformer in the network. The provisioning of new services will be stewarded by the rise of new network control and management solutions in WDM networks which will facilitate and automate that task (Brunner et al., 2004). Either coupling or decoupling can be induced in the predicament of routing and assignment in Routing and Wavelength Assignment (RWA) (Ramaswami and Sivarajan 1995; Xu et al., 2000). The problem of increasing the number of accepted connections in the RWA was dealt by the work in (Thiongane, 2011). The literatures for the analytical close for the evaluation of performance of the RWA problem are very few because most of the existing works related to these are just simulations. A wavelengthdivision multiplexing with orthogonal-frequencydivision-multiplexing passive-optical network architecture with centralized light wave sources and polarization shift keying multicast overlay was discussed in (Liu et al., 2010). The $10 \mathrm{Gbps}$ 16QAM-OFDM point to point signal, $2.5 \mathrm{Gbps}$ multicast signal and $2.5 \mathrm{Gbps}$ On-Off Keying (OOK) upstream signal were experimented over $25 \mathrm{~km}$ standard single mode fiber. The crosstalk between the downstream signals was eliminated in the receiver by employing low pass filter. The power penalty for both up and downstream multicast signal was analyzed. The static multicast advance reservation problem for all-optical wavelength-routed WDM networks was proposed in the work by (Charbonneau and Vokkarane, 2012). Under the advanced reservation traffic model, connection requests specify their start time to be some time in the future and also specifies their holding times. The set of advance reservation requests was known ahead of time. A theoretical lower bound on the number of wavelengths required was worked out. It was observed that the multipath heuristics approach was close to optimal results.

The work presented in (Sridharan and Sivarajan, 2004; Ramesh et al., 2000; Ramamurthy and Mukherjee, 1998; Xiong et al., 2003; Cerutti et al., 2005). The work presented in (Pitchumani et al., 2004; Chen et al., 2001) deals with the classes of connections which are differentiated, by the admission control policies, by their blocking probabilities. Also the work in (Tan et al., 2006) handles the provision of different QoS to the various priority classes in a WDM optical burst switching networks. An extension to the unicast RWA problem, the Weighted Multicast Routing and Wavelength Assignment (WMRWA) problem has been addressed in this study. The connection between the source and target is constructed with the flock of links and wavelengths which are found by WMRWA, with the multicast calls and the restricted number of wavelengths. Using this technique, the network can settle down to an optimized solution with minimal delay by using the random weight method. This minimizes the call blocking probability along with a numerical increase in the multicast calls that are admitted at a time. With the provisioning of variable wavelengths in a single light path can serve more connection requests at a time in the very same light path. Due to the importance of the wavelength assignments in optical network, the proposed work focuses on increasing the network wavelength availability with the QoS parameters considered.

The rest of the study is organized as follows: The model of the network under deliberation with the presented work and the detailed depiction of the issues are given in section 2. Section 3 contains the explanation for the WMRWA approach. The experimental setup and the performance evaluation using our proposed approach are crafted in the section 4. Section 5 concludes with certain remarks.

\section{MATERIALS AND METHODS}

The WDM based network model and the problem statement for the presented work is detailed in this section.

\subsection{Network Model}

The minimization of call blocking probability and the maximization of the network capacity is aimed by the assignment of wavelength, with the currently given state of a network and the new arrival request. Hence we focus on the fresh league of ideas which solely undertakes the state of the network in the race of minimizing the call blocking probability.

An undirected graph $\mathrm{G}(\mathrm{V}, \mathrm{E})$, represents the WDM mesh network (network topology is bidirectional) under scrutiny, where the group of network nodes posing the Optical Cross-Connects (OXCs) is given by $\mathrm{V}$ and the group of network links posing optical fibres are being represented by $\mathrm{E}$. Let $\mathrm{n}$ and $\mathrm{m}$ be the number of nodes and links in the network. We also assume that $\mathrm{W}$ represents the wavelengths which are used in each link and we also assume that a link can accommodate one optical fibre. Each wavelength used is related to the cost $C(i, j)$ which characterizes each link $(i, j)$. Also we imagine that in the bidirectional propagation a link fault may disrupt the connection in either of the direction. The nomenclature and notations used in our proposed models are as follows: 


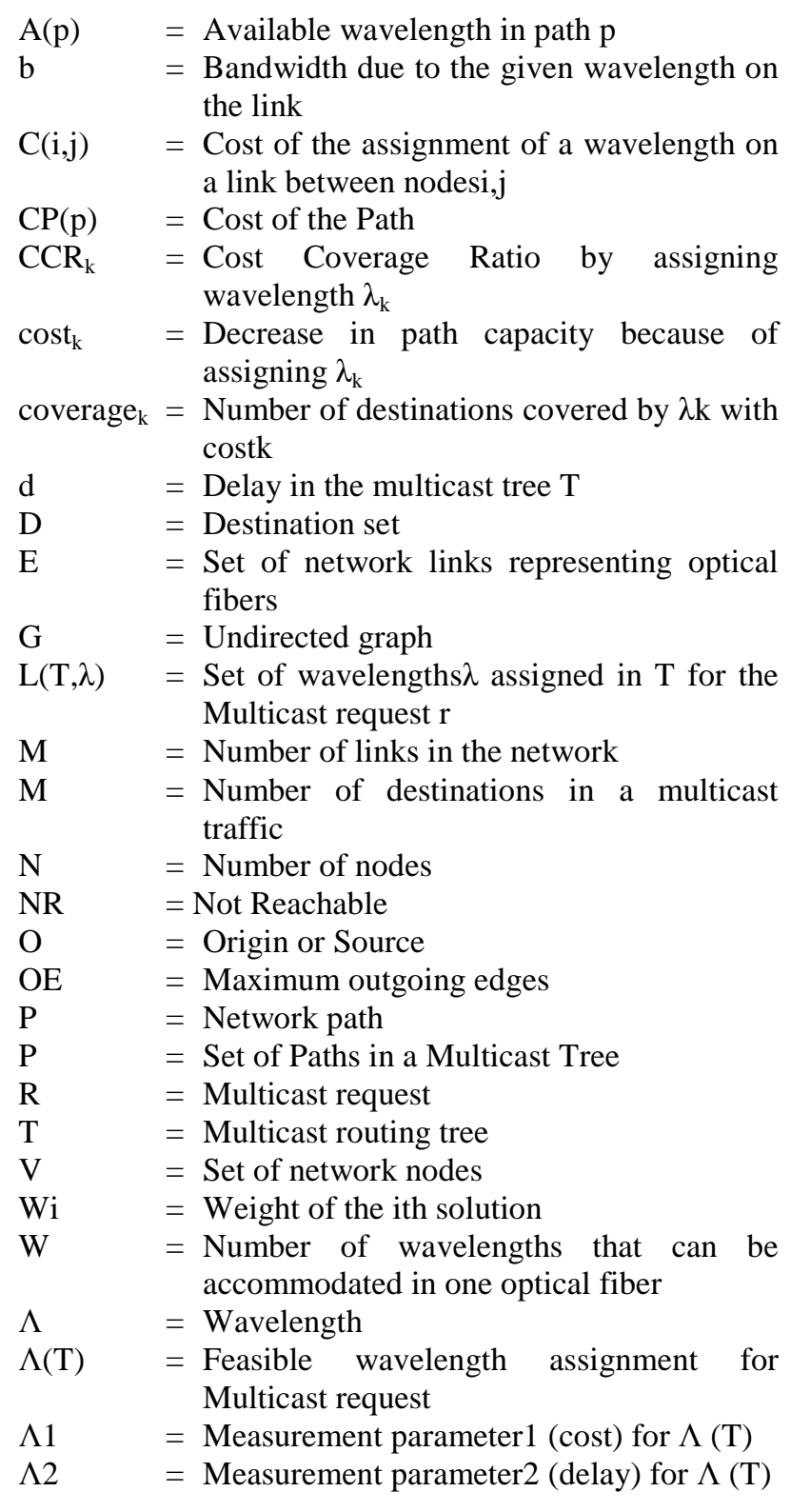

The performance parameters such as the network delay $\mathrm{dm}$ and its tolerant are the vital QoS requirements in multicast application. Few applications need a much decreased delay value but others might be capable of accepting up to a tolerance value. The cost of a path $\mathrm{p}$, says CP (p), is computed as sum of the link costs in the path and is given as Equation 1:

$$
\mathrm{CP}[\mathrm{p}]=\sum_{(\mathrm{i}, \mathrm{j}) \in \mathrm{P}} \mathrm{C}(\mathrm{i}, \mathrm{j})
$$

\subsection{Problem Statement}

The problem is formally defined as follows: A traffic matrix represents the traffic across the nodes in the network due to multicast requests. The summation of the path capacities for all $\mathrm{M}$ paths is defined to be the path capacity of the multicast.

Consider the new arrival multicast request, $r=\{0, D$, $\mathrm{d}, \mathrm{T}$ \} for each multi spanning tree, where $\mathrm{o}, \mathrm{D}, \mathrm{d}$ and $\mathrm{T}$ denote the multicast origin, destination set, delay and multicast routing tree respectively. According to wavelength assignment, $\mathrm{L}(\mathrm{T}, \lambda)$ is a set ofwavelengths $(\lambda)$ given for the request ' $r$ '.

Wavelength assignment for maximizing network capacity for the given multicast request must choose $\Lambda^{*}(\mathrm{~T})$ such that Equation 2:

$|\{(\lambda, \mathrm{p}): \mathrm{p} \in \mathrm{P},(\lambda \in \mathrm{A}(\mathrm{p}) \cap \mathrm{L}(\mathrm{T}, \lambda)) \neq \varphi\}|$

Is the most minimal in all possible wavelength assignments for $\mathrm{T}$.

The given WMRWA algorithm aims to meet the optimized solution from the variety of probably appealing solutions generated by (2) as:

$\sum_{\mathrm{i}=1}^{\mathrm{n}}\left(\mathrm{w}_{\mathrm{i}} * \Lambda_{1}^{*}\right)+\left(\left(1-\mathrm{w}_{\mathrm{i}}\right) * \Lambda_{2}^{*}\right)$

where, $\Lambda 1^{*}$ and $\Lambda 2 *$ are the two measurement parameters such as cost and delay and wi is the weight component with the value from 0 to 1 trading between cost and delay.

\subsection{Proposed Work}

The usage of the Greedy algorithm, along with our proposed methods manoeuvring the maximization of the network capacity in the multicast wavelength assignment problem and is discussed in this section.

\subsection{Existing Methodologies}

From the available wavelengths, the wavelength with the minimum Wavelength Cost Ratio (WCR) is simply chosen by each target according to the Static Cost Greedy (SCG) algorithm given in (Zang et al., 2000). Then, according to the WCR the wavelengths are arranged in the ascending order and thus each wavelength is taken separately and assigned to all reachable targets, until the exhaustion of all wavelengths in the sorted stack. 
Time complexity of the SCG algorithm is O $(|\mathrm{P}| \mathrm{NW}+\mathrm{W} \log (\mathrm{W}))$ where, $\mathrm{W}$ is the Maximum number of wavelengths in each link, $\mathrm{N}$ denotes Number of nodes and $\mathrm{P}$ represents Path Capacity

The efficiency of this algorithm is limited because of the reason that only very few destinations could be reached. Applying this algorithm might reduce the total path capacity by assigning a wavelength which is normally calculated by figuring out the number of potential connections present in the traffic matrix, with a unit decrease in path capacity. The best wavelength cost ratio is calculated and picked.

In contrast to the SCG, the Dynamic Cost Greedy (DCG) algorithm stated in (Zang et al., 2000) upgrades the WCR after the usage of each wavelength and also it first chooses the wavelength with minimal WCR to accommodate all possible targets, thus the estimation of cost for each of the remaining wavelength becomes better. $\mathrm{O}(|\mathrm{P}| \mathrm{NW} 2)$ is the time complexity, as a result of the Dynamic Cost Greedy algorithm. The construction of spanning trees for the graph without duplication, via elementary tree transformations, was done by the Mayeda and Seshu algorithm (Mayeda and Seshu, 1995). The edges of the graph are removed by means of various combinations and the multiple spanning trees are constructed. The series of small and regular interval time for payback $\mathrm{O}(\mathrm{nm})$ is required for the algorithm to operate and also $\mathrm{O}(\mathrm{nm})$ space is essential.

\subsection{Proposed Weighted Multicast Routing and Wavelength Assignment (WMRWA) Considering Delay}

The wave length assignment method which takes the delay (QoS parameter) into account for assigning wavelength for multicast in WDM all-optical networks is put forth in our study. With the reduction of delay in communication transmission the call blocking probability gets minimized, hence it focuses on the newer type of objective which takes the network state in mind. The procedure for applying MRWA algorithm is shown as below:

Algorithm assignOptimalWavelength ( )

\{

//Input:Graph $\mathrm{G}=(\mathrm{V}, \mathrm{E})$ with wavelength capacity at each link, $\Lambda=\left\{\lambda_{1}, \lambda_{2}, \ldots, \lambda_{\mathrm{W}}\right\}$

//Output: Optimum solution with allotted wavelength for the demand

$/ / \mathrm{D}_{\mathrm{k}}-$ Destinations reachable using $\lambda_{\mathrm{k}}$

// W - maximum number of wavelength in each link

$/ / \mathrm{CCR}_{\mathrm{k}}$ - cost coverage ratio with $\lambda_{\mathrm{k}}$

\section{\{}

$\mathrm{T}=$ constuctSpanningTree $(\mathrm{G}) / /$ UseMayeda and Seshu algorithm $T=\left\{T_{1}, T_{2}, \ldots T_{m}\right\}$

// $\mathrm{P}=\left\{\mathrm{P}_{1}, \mathrm{P}_{2}, \mathrm{P}_{\mathrm{i}}, \ldots \mathrm{P}_{\mathrm{n}}\right\}$ is the set of paths to the destinations

$\mathrm{D}=\left\{\mathrm{d}_{1}, \mathrm{~d}_{2}, \ldots, \mathrm{d}_{\mathrm{r}}\right\} \quad / /$ set of destinations

initWavelength $(\Lambda) ; / / \Lambda=\left\{\lambda_{1}, \lambda_{2}, \ldots, \lambda_{\mathrm{W}}\right\}$

for each spanning Tree $T_{r}$ in $T$

for each path $P_{i}$ in $T_{r}$

for each wavelength $\lambda_{k}$

\{

$\operatorname{cost}_{\mathrm{k}}=$ computeCostToDest $(\mathrm{D})$

//Use Dynamic Cost Greedy algorithm (Zang et al., 2000)

$\mathrm{CCR}_{\mathrm{k}}=\operatorname{cost}_{\mathrm{k}} /$ coverage $_{\mathrm{k}}$

\}

$\mathrm{CCR}=$ sort $(\mathrm{CCR}) / /$ For choosingCCR $\mathrm{k}_{\mathrm{k}}$ of $\lambda_{\mathrm{k}}$ in increasing order

Find $D_{k}$

Find all $\mathrm{D}-\left\{\mathrm{D}_{\mathrm{k}}\right\}$ using $\mathrm{CCR}_{\mathrm{k}+1} \ldots \mathrm{CCR}_{\mathrm{W}}$

$\mathrm{P}=\mathrm{P}-\left\{\mathrm{P}_{\mathrm{i}}\right\}$

//Finding delay to $\mathrm{D}$ through the spanning tree $\mathrm{T}_{\mathrm{r}}$

$\mathrm{TDr}=\operatorname{calcDelay}\left(\mathrm{T}_{\mathrm{r}}\right)$

// Finding optimum solution using WMRWA.

$\mathrm{W}_{\mathrm{k}}=$ Randomly Assign Weights for each $\mathrm{TD}_{\mathrm{k}}, \mathrm{CCR}_{\mathrm{k}}$; findOptimalSpanningTree (TDOW, CCROW, w);

//OW-Optimal set of wavelengths

\}

This algorithm dynamically calculates the Cost Coverage Ratio (CCR) for each spanning tree using the formula Equation 4:

$$
\mathrm{CCR} \frac{\cos t_{k}}{\text { Coverage }_{k}}
$$

where, $\operatorname{cost}_{\mathrm{k}}$ is the decrease in path capacity because of assigning $\lambda_{\mathrm{k}}$ and coverage $\mathrm{k}_{\mathrm{k}}$ is the number of destinations covered by $\lambda_{\mathrm{k}}$ with $\operatorname{cost}_{\mathrm{k}}$. Also ' $\mathrm{d}$ 'is the delay to reach all the destinations through the optimal spanning tree. The delay in each path is obtained using Equation 5:

$$
\mathrm{d}=\frac{1}{\mathrm{bw}}+\frac{\text { dist }}{\mathrm{c}}
$$

where, $\mathrm{d}$ is the delay due to the transmission and propagation delay, $\mathrm{c}$ is the speed of light. In general, as our WMRWA algorithm uses multiple spanning trees and multiple parameters, it is seeking for optimal solution. 


\subsection{Experimental Setup}

The experimental set up for the evaluation of the presented work and the numerical results obtained are given in this section. The experimental setup and the other simulation model parameters that were observed in the system design are given followed by the comparison between the two Greedy algorithms and the proposed WMRWA (from section 3) approach.

The Cost Coverage Ratio (Equation 3) is the indirect measurement of the blocking probability, which is defined as the ratio of the number of blocked connections to the total number of connections injected into a network.

We conduct our experiments on two sample networks: NET1 that has 6 nodes and 10 bidirectional links as shown in Fig. 1a and for NET2 (COST239 network) with 11 nodes and 23 bidirectional links that has been shown in Fig. 1b Each link in these networks represents two unidirectional fiber links in opposite directions. Available wavelength in each of the link is also shown in the figure. Throughout our experiments, we use the following parameters:

i. N-Network size

ii. W-Maximum number of available wavelengths on each link

iii. OE-Maximum outgoing degree in each node

iv. M-Number of destinations in the multicast session

With the help of the above parameters, the steps given below are adapted for creating the setup:

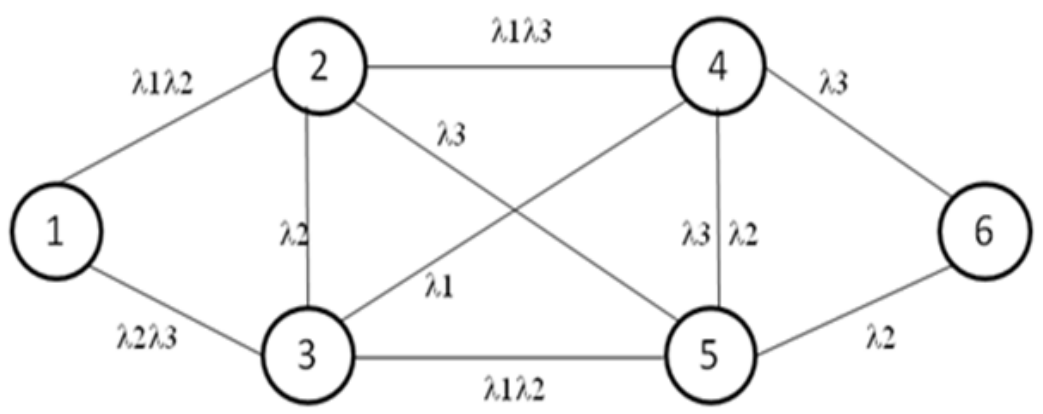

(a)

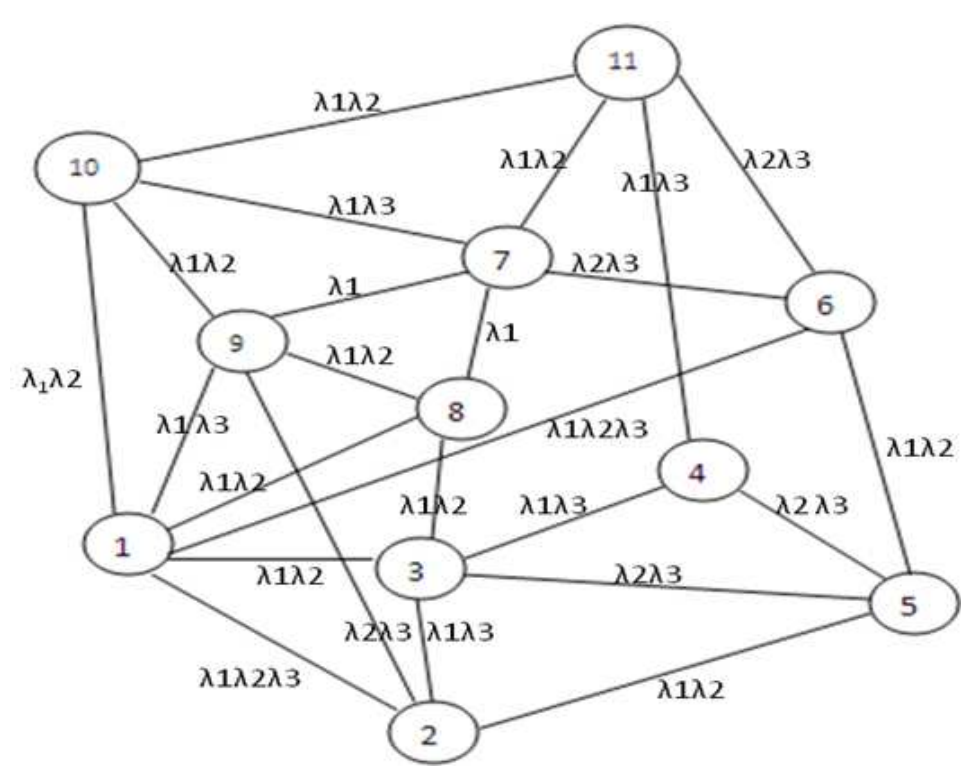

(b)

Fig. 1. (a) Experimental sample network setup (b) For 6 nodes with 10 bidirectional links and (b) for standard COST 239 Network with 11 nodes having 23 bidirectional links 
With the help of the above parameters, the steps given below are adapted for creating the setup:

- Generate a network of $\mathrm{n}$ nodes in random. Out represents the out degree of each node i. The generation of $\mathrm{Out}_{\mathrm{i}}$ is randomly selected from node $\mathrm{i}$ to remaining nodes, since it is uniformly distributed in $[1, \ldots \mathrm{OE}]$. The number of available wavelengths for each link, are uniformly distributed in $[1, \ldots \mathrm{W}]$

- In multicast session one node is selected to be the origin and the remaining ' $\mathrm{n}$ ' nodes to be the target. When we run the single-source shortest-path algorithm from the origin to all targets, a multicast tree is obtained. However there are cases that not every target has a path from the origin. In that regard, we have to revoke this instance and invoke another network spanning tree

- For the reference multicast, invoke all possible spanning trees

Soon after establishing the network models, we induce the simulation of the proposed algorithm and the SCG and DCG algorithms. Then the following steps are carried out:

- Take into account, a multicast session comprising node 1 as origin and other nodes as targets

- Build and establish MMST

- Build and establish the shortest path for every spanning tree to reach the targets
- Compute Cost Coverage Ratio $\mathrm{CCR}_{\mathrm{k}}=\operatorname{cost}_{\mathrm{k}} /$ coverage $_{\mathrm{k}}$ and delay for every path, where $\operatorname{cost}_{\mathrm{k}}$ is the decrease in network capacity (while using $\lambda_{\mathrm{k}}$ ) and coverage $\mathrm{k}_{\mathrm{k}}$ is the number of reachable targets using a specific $\lambda_{\mathrm{k}}$ as shown in Table 1

- Sort out the spanning trees with minimum delay, as well as the spanning trees with minimum Cost Coverage Ratio and unearth the optimal spanning tree

The process of wavelength assignment (steps 1 to 7 ) is repeated for all of the multicast trees and the total network capacity decrease in each case is calculated. The results are tabulated in Table 2 for the cases of 6 nodes and COST 239 network models. Total network capacity decrease is the decrease of capacity (i.e., how many future requests are unable to assign the wavelength) when assigning a particular wavelength to each destination. Based on the total network capacity decrease, the tree that have the lower value will be considered as the optimum route and that wavelength assignment will provide minimum call blocking probability.

\section{RESULTS AND DISCUSSION}

Table 1a and 1b show reachable destinations with available wavelengths in multiple spanning trees with our WMRWA approach for 6 Nodes Networks and COST239 Networks respectively.

Table 1a. Reachable destinations with available wavelengths in multiple spanning trees with our wmrwa for 6 nodes networks

\begin{tabular}{|c|c|c|c|c|c|c|}
\hline \multirow{2}{*}{$\begin{array}{l}\text { N/W with 6- Nodes } \\
\text { To destination }\end{array}$} & \multicolumn{6}{|c|}{ Spanning Trees (ST) reachable using wavelength $(\lambda)$} \\
\hline & ST 1 & ST 2 & ST 3 & ST 4 & ST 5 & ST 6 \\
\hline $\begin{array}{l}2 \\
3\end{array}$ & $\begin{array}{l}\lambda_{1} \\
\lambda_{3}\end{array}$ & $\begin{array}{l}\lambda_{3} \\
\lambda_{2}\end{array}$ & $\begin{array}{l}\lambda_{2} \\
\lambda_{1}\end{array}$ & $\begin{array}{l}\lambda_{2} \\
\lambda_{3}\end{array}$ & $\begin{array}{l}\lambda_{1} \\
\lambda_{3}\end{array}$ & $\begin{array}{l}\lambda_{1} \\
\lambda_{3}\end{array}$ \\
\hline 4 & $\lambda_{2}$ & $\lambda_{2}^{2}$ & NR & $\lambda_{1}^{2}$ & $\lambda_{2}$ & $\lambda_{2}$ \\
\hline 5 & $\lambda_{2}$ & NR & NR & NR & $\lambda_{1}$ & $\lambda_{2}$ \\
\hline 6 & $\lambda_{2}$ & $\lambda_{2}$ & NR & NR & $\lambda_{2}$ & NR \\
\hline
\end{tabular}

Table 1b. Reachable destinations with available wavelengths in multiple spanning trees with our wmrwa for cost 239 Networks

\begin{tabular}{|c|c|c|c|c|c|c|c|c|c|c|c|}
\hline \multirow{2}{*}{$\begin{array}{l}\text { COST } 239 \mathrm{~N} / \mathrm{W} \\
\text { To Destination }\end{array}$} & \multicolumn{11}{|c|}{ Spanning Trees (ST) reachable using wavelength $(\lambda)$} \\
\hline & ST1 & ST2 & ST3 & ST4 & ST5 & ST6 & ST7 & ST8 & ST9 & ST10 & ST11 \\
\hline 2 & $\lambda_{2}$ & $\lambda_{1}$ & $\lambda_{3}$ & $\lambda_{3}$ & $\lambda_{1}$ & $\lambda_{2}$ & $\lambda_{3}$ & $\lambda_{2}$ & $\lambda_{2}$ & $\lambda_{2}$ & $\lambda_{2}$ \\
\hline 3 & $\lambda_{2}^{2}$ & $\lambda_{1}$ & $\lambda_{1}$ & $\lambda_{3}$ & $\lambda_{1}$ & $\lambda_{2}^{2}$ & $\lambda_{3}$ & $\lambda_{2}$ & $\lambda_{2}^{2}$ & $\lambda_{2}$ & $\lambda_{2}^{2}$ \\
\hline 4 & $\lambda_{2}^{2}$ & $\lambda_{1}$ & $\lambda_{1}$ & $\lambda_{3}$ & $\lambda_{1}$ & $\lambda_{2}^{2}$ & $\lambda_{1}$ & $\lambda_{2}$ & $\lambda_{2}$ & $\lambda_{2}$ & $\lambda_{2}^{2}$ \\
\hline 5 & $\lambda_{1}$ & $\lambda_{1}$ & $\lambda_{1}$ & $\lambda_{3}$ & $\lambda_{1}$ & $\lambda_{1}$ & $\lambda_{1}$ & $\lambda_{1}$ & $\lambda_{1}$ & $\lambda_{1}$ & $\lambda_{1}$ \\
\hline 6 & $\lambda_{2}$ & NR & $\lambda_{1}$ & $\lambda_{1}$ & $\lambda_{2}$ & NR & $\lambda_{1}$ & $\lambda_{2}$ & $\lambda_{2}$ & $\lambda_{2}$ & $\lambda_{2}$ \\
\hline 7 & $\lambda_{2}$ & $\lambda_{2}$ & $\lambda_{1}$ & $\lambda_{3}$ & $\lambda_{3}$ & $\lambda_{2}$ & $\lambda_{1}$ & $\lambda_{2}$ & $\lambda_{2}$ & $\lambda_{2}$ & $\lambda_{2}$ \\
\hline 8 & NR & NR & NR & NR & NR & NR & $\lambda_{1}$ & $\lambda_{2}$ & NR & NR & NR \\
\hline 9 & NR & NR & $\lambda_{1}$ & NR & NR & NR & $\lambda_{1}$ & $\lambda_{2}$ & $\lambda_{2}$ & NR & NR \\
\hline 10 & NR & NR & $\lambda_{1}$ & NR & NR & NR & $\lambda_{1}$ & $\lambda_{2}$ & $\lambda_{2}$ & $\lambda_{2}$ & NR \\
\hline 11 & $\lambda_{2}$ & $\lambda_{2}$ & $\lambda_{1}$ & $\lambda_{3}$ & $\lambda_{3}$ & $\lambda_{2}$ & $\lambda_{3}$ & $\lambda_{2}$ & $\lambda_{2}$ & $\lambda_{2}$ & $\lambda_{1}$ \\
\hline
\end{tabular}


Table 2. Comparison of our proposed WMRWA with SCG and DCG in terms of CCR and Delay

\begin{tabular}{|c|c|c|c|c|c|c|c|c|c|c|c|}
\hline \multirow[b]{2}{*}{ Sample network } & \multirow[b]{2}{*}{ Source } & \multicolumn{3}{|l|}{ SCG } & \multicolumn{3}{|l|}{ DCG } & \multicolumn{4}{|c|}{ Proposed WMRWA } \\
\hline & & $\begin{array}{l}\text { Destinations } \\
\text { reachable }\end{array}$ & CCR & $\begin{array}{l}\text { Delay } \\
(\mathrm{ms})\end{array}$ & $\begin{array}{l}\text { Destinations } \\
\text { reachable }\end{array}$ & CCR & $\begin{array}{c}\text { Delay } \\
(\mathrm{ms})\end{array}$ & $\begin{array}{l}\text { Spanning } \\
\text { Tree No. }\end{array}$ & $\begin{array}{l}\text { Destinations } \\
\text { reachable }\end{array}$ & CCR & $\begin{array}{l}\text { Delay } \\
(\mathrm{ms})\end{array}$ \\
\hline 6 Nodes & 1 & $2,3,4$ & 3 & 0.58 & $2,3,4,5,6$ & 2.4 & 0.28 & I & $2,3,4,5,6$ & 2.40 & 0.28 \\
\hline \multirow[t]{5}{*}{ Mesh Network } & & & & & & & & II & $2,3,4,6$ & 2.50 & 0.18 \\
\hline & & & & & & & & III & 2,3 & 4.00 & 0.15 \\
\hline & & & & & & & & IV & $2,3,4$ & 2.33 & 0.20 \\
\hline & & & & & & & & V & $2,3,4,5,6$ & 2.20 & 0.33 \\
\hline & & & & & & & & VI & $2,3,4,5,6$ & 2.40 & 0.23 \\
\hline \multirow[t]{11}{*}{$\begin{array}{l}\text { COST239 Network } \\
\text { with } 11 \text { Nodes }\end{array}$} & 1 & $\begin{array}{l}2,3,4,5,6, \\
7,8,9,10,11\end{array}$ & 1.75 & 1.23 & $\begin{array}{l}2,3,4,5,6 \\
7,8,9,10,11\end{array}$ & 1.4 & 0.8 & I & $2,3,4,5,6,7,8,9,10,11$ & 1.40 & 0.80 \\
\hline & & & & & & & & II & $2,3,4,5,7,11$ & 1.83 & 0.50 \\
\hline & & & & & & & & III & $2,3,4,5,6,7,9,10,11$ & 1.67 & 0.83 \\
\hline & & & & & & & & IV & $2,3,4,5,6,7,10,11$ & 1.86 & 0.30 \\
\hline & & & & & & & & $\mathrm{V}$ & $2,3,4,5,6,7,10,11$ & 2.00 & 0.52 \\
\hline & & & & & & & & VI & $2,3,4,5,7,10,11$ & 1.83 & 0.35 \\
\hline & & & & & & & & VII & $2,3,4,5,6,7,11$ & 1.71 & 0.40 \\
\hline & & & & & & & & VIII & $2,3,4,5,6,7,8,9,10,11$ & 1.33 & 0.55 \\
\hline & & & & & & & & IX & $2,3,4,5,6,7,9,10,11$ & 1.56 & 0.50 \\
\hline & & & & & & & & $\mathrm{X}$ & $2,3,4,5,6,7,10,11$ & 1.63 & 0.45 \\
\hline & & & & & & & & XI & $2,3,4,5,6,7,11$ & 1.57 & 0.45 \\
\hline
\end{tabular}

In the first case only the spanning tress 1 and 5 are the valid ones in the sense that they are covering all the intended destinations. Similarly for the second case spanning trees 7 and 8 are covering all the destinations. From these spanning trees only the appropriate one has to be selected with respect to cost and delay thereby increasing the network capacity. From Table 2, for the sample network with 6 nodes case, one can find that the $\mathrm{CCR}$ value obtained in spanning tree $\mathrm{V}$ is the lowest one but the delay for spanning tree $\mathrm{V}$ is too high. Hence spanning tree $\mathrm{V}$ seems to be a better choice while compared with SCG and DCG technique. But according to the QoS parameter delay, spanning tree III has a lower value. In the goal of finding an optimum solution which has a lower value for both CCR and delay, one can select spanning tree VI that has CCR value with 2.4 and delay as 0.23 . It is clear that, the system with DCG technique will settle in the first spanning tree having CCR is 2.4 (same as that of our optimum solution) and delay is 0.28 (which is higher than optimum solution) i.e., a local minima, where as our proposed technique has a delay of 0.23 with same CCR value i.e., a global minima.

For Cost239 network, the DCG will settle at the local minima solution of CCR as 1.4 and delay as 0.80 $\mathrm{ms}$, where as our proposed method searches globally on all possible spanning trees and coming up with a solution of CCR as 1.33 and delay as $0.55 \mathrm{~ms}$ (in spanning tree VIII). Hence it is clear that, our method produces a better output compared to other previous methods SCG and DCG. Depending on the application, the two parameters CCR and delay can be traded-off. The novelty of the work lies in the construction of multiple minimum spanning trees for the further considerations of the QoS constraints.

\section{CONCLUSION}

While many of the current Routing and Wavelength Assignment algorithms takes only the routing and available wavelengths into account, the proposed work pays rather great concern towards the different Quality of Service (QoS) parameters such as delay, along with its primary goal of minimizing the call blocking probability. The performance of our proposed method is satisfying due to the fact that it looks for the optimized solution via multiple spanning trees. Also the performance measurement parameters such as cost and delay can be traded off depending on the application using $\mathrm{CD}=\mathrm{C} \alpha+$ $(1-\alpha) * \mathrm{D}$ where, $\mathrm{C}$ is the Cost and D is the Delay. Our future work aim's at setting an objective function for Cost Coverage Ratio and delay with minimum computational complexity.

\section{ACKNOWLEDGEMENT}

The researchers duly acknowledge the support provided by the Management and Principal of Mepco Schlenk Engineering College and P.S.R. Engineering College by means of providing all the research facilities. 


\section{REFERENCES}

Brunner, M., H. Mouftah and M. Ulema, 2004. Management of optical networks and services. IEEE Commun. Mag., 42: 34-35. DOI: 10.1109/MCOM.2004.1262159

Cao, Y. and $\mathrm{O}$. $\mathrm{Yu}, 2005$. QoS-guaranteed routing and wavelength assignment for group multicast in optical WDM networks. Proceedings of the Conference on Optical Network Design and Modeling, Feb. 7-9, IEEE Xplore Press, pp: 175184. DOI: 10.1109/ONDM.2005.1426999

Cerutti, I., S. Pitchumani and A. Fumagalli, 2005. A threshold-based blocking differentiation mechanism for networks with wavelength continuity constraint. Proceedings of 7 th International Conference Transparent Optical Networks, Jul. 3-7, IEEE Xplore Press, pp: 179-182. DOI: 10.1109/ICTON.2005.1505781

Charbonneau, N. and V.M. Vokkarane, 2012. Static routing and wavelength assignment for multicast advance reservation in all-optical wavelength-routed WDM networks. IEEE/ACM Trans. Network., 20: 1-14. DOI: 10.1109/TNET.2011.2175007

Chen, K.S., C.P. Yu, C. Yu and N.F. Huang, 2004. Provisioning multicast QoS for WDM-based optical wireless networks. J. Comput. Commun., 27: 10251035. DOI: 10.1016/j.comcom.2004.01.025

Chen, Y., M. Hamdi and D.H.K. Tsang, 2001. Proportional QoS over WDM networks: Blocking probability. Proceedings of the 6th IEEE Symposium on Computers and Communications, Jul. 3-5, IEEE Xplore Press, Hammamet, pp: 210215. DOI: 10.1109/ISCC.2001.935377

Coiro, A., M. Listanti, A. Valenti and F. Matera, 2011. Power aware routing and wavelength assignment in multi-fiber optical networks. IEEE/OSA Journal of Optical Communications. and Networking, 3: 816-829. DOI: 10.1364/JOCN.3.000816

Fumagalli, A. and M. Tacca, 2001a. Optimal design of optical ring networks with Differentiated Reliability (DiR). Proceedings of the International Workshop Quality of Service in Multiservice IP Networks, Jan. 24-26, Springer Berlin Heidelberg, Rome, Italy, pp: 299-314. DOI: 10.1007/3-54044554-4_20
Fumagalli, A. and M. Tacca, 2001b. Differentiated reliability (DiR) in WDM rings without wavelength converters. Proceeding of the IEEE International Conference on Communications, (ICC, 01) Helsinki, Finland, pp: 2887-2891. DOI: 10.1109/ICC.2001.936677

Golmie, N., T.D. Ndousse and D.H. Sue, 2000. A differentiated optical services model for WDM networks. IEEE Commun. Mag., 38: 68-73. DOI: 10.1109/35.819898

Liu, B., X. Xin, L. Zhang, J. Yu and Q. Zhang et al., 2010. A WDM-OFDM-PON architecture with centralized light wave and PolSK-modulated multicast overlay. Optics Express, 18: 2137-2143. DOI: 10.1364/OE.18.002137

Mayeda, W. and S. Seshu, 1965. Generation of trees without duplications. IEEE Trans. Circ. Theory, 12: 181-185. DOI: 10.1109/TCT.1965.1082432

Pitchumani, S., I. Cerutti and A. Fumagalli, 2004. Threshold-based blocking differentiation in circuit-switched WDM networks. Proceedings of the Global Telecommunications Conference, Nov. 29-Dec. 3, IEEE Xplore Press, pp: 995-999. DOI: 10.1109/GLOCOM.2004.1378108

Ramamurthy, R. and B. Mukherjee, 1998. Fixedalternate routing and wavelength conversion in wavelength-routed optical networks. Proceedings of the IEEE The Bridge to Global Integration Global Telecommunications Conference, Nov. 812, IEEE Xplore Press, Sydney, NSW, pp: $2295-$ 2302. DOI: 10.1109/GLOCOM.1998.775940

Ramaswami, R. and K.N. Sivarajan, 1995. Routing and wavelength assignment in all-optical networks. IEEE/ACM Trans. Netw., 3: 489-500. DOI: 10.1109/90.469957

Ramesh, S., G.N. Rouskas and H.G. Perros, 2002. Computing blocking probabilities in multiclass wavelength-routing networks with multicast calls. IEEE J. Selected Areas Commun., 20: 89-96. DOI: 10.1109/49.974664

Sridharan, A. and K.N. Sivarajan, 2004. Blocking in alloptical networks. IEEE/ACM Trans. Netw., 12: 384397. DOI: 10.1109/TNET.2004.826251

Tan, C.W., G. Mohan and J.C.S. Lui, 2006. Achieving multi-class service differentiation in WDM optical burst switching networks: A probabilistic preemptive burst segmentation scheme. IEEE J. Selected Areas Commun., 24: 106-119. DOI: 10.1109/JSAC.2006.258227 
Thiongane, B., 2011. Lagrangean decomposition/relaxation for the routing and wavelength assignment problem. Networks, 59: 226239. DOI: $10.1002 /$ net.20437

Xiong, J., W. Gong and C. Qiao, 2003. An effective method for blocking performance analysis of WDM all-optical networks. J. Light W. Technol., 21: 14211434. DOI: 10.1109/JLT.2003.810083

$\mathrm{Xu}$, S., L. Li, S. Wang and C. Chen, 2000. Wavelength assignment for dynamic traffic in WDM networks. Proceedings of the IEEE International Conference on Networks, (ICN, 00), IEEE Xplore Press, pp: 375-379. DOI: 10.1109/ICON.2000.875817
Zang, H., J.P. Jue and B. Mukherjee, 2000. A review of routing and wavelength assignment approaches for wavelength-routed optical WDM networks. SPIE Opt. Netw. Mag., 1: 47-60.

Zhang, H. and E.W. Knightly, 1999. Integrated and differentiated services for the internet. IEEE Netw., 13: 7-7. DOI: 10.1109/MNET.1999.793685 